\title{
Isolement de souches atypiques \\ de Microsporum canis dans la région parisienne
}

\author{
par E. RIVALIER et G. BADILLET \\ [Laboratoire Sabouraud (service du $\mathrm{P}^{\mathrm{r}}$ Degos) et Laboratoire Alibert, \\ (service du $P^{r}$ Duperrat), Hôpital Saint-Louis, rue Bichat, 75 - Paris-10]
}

\section{Résumé}

205 souches de Microsporum ont été isolées à Paris en dixhuit mois, d'octobre 1966 à mars 1968. Sur ce nombre, 23 souches, toutes identiques, présentaient des caractères différents des souches habituelles de $M$. canis: cultures blanches, pulvérulentes ou finement duveteuses, à envers jamais coloré en jaune ou en orangé ; à l'examen microscopique, présence de très nombreuses microconidies périformes et de macroconidies de type $M$. canis, mais beaucoup moins nombreuses que dans les souches habituelles. Tous les autres caractères sont ceux de Microsporum canis. On propose le nom de Microsporum canis var. pulverulentum. La connaissance de ces souches présente, outre un intérêt purement mycologique, une grande importance pour la prophylaxie des teignes en raison de la confusion possible avec Microsporum audouini

\section{Summary}

In 18 months from October 1966 to March 1968, 205 strains of Microsporum have been isolated in Paris. Among them, 23 strains showed features different from the usual strains of Microsporum canis: powdery or finely velvety white cultures without yellow or orangecoloured reverses; microscopically, very many clavate microconidia, and macroconidia but far less numerous than in usual strains. The name of Microsporum canis var. pulverulentum is proposed. The discovery of these strains presents, beside a purely mycological interest, a great importance because of the possible mistaking with Microsporum audouini.

Annales de Parasitologie humaine et comparée (Paris), t. 44, 1969, $\mathrm{n}^{\circ} 3$ 
D'octobre 1966 à mars 1968, soit en un an et demi, nous avons vu à Paris 205 malades atteints de microspories. La plupart des cultures obtenues ont été facilement identifiées à Microsporum canis. Cependant, 23 de ces cultures de Microsporum nous ont posé des problèmes d'identification. Au fur et à mesure de leur isolement, il nous a paru que ces 23 souches présentaient un ensemble homogène, avec des caractères culturaux particuliers. C'est pourquoi nous les avons réunies dans ce travail.

\section{Etude clinique.}

Le tableau ci-contre résume nos observations cliniques:

Il en ressort un certain nombre de notions :

$1^{\circ}$ Assez souvent, il s'agit de petites épidémies familiales.

C'est le cas de la famille Co..., où la mère et un enfant ont été atteints, de la famille Bar... où quatre frères et sœurs ont présenté des herpès circinés, de la famille Thi... où trois enfants et la mère ont été contaminés, de la famille Gar... où trois frères ont été atteints de teignes ou d'herpès circinés, enfin, de la famille Lac... où trois scurs ont également été atteintes de teignes ou d'herpès circinés.

Plus rarement, il s'agit de cas isolés chez des adultes, telle $\mathbf{M}^{\text {He }}$ Bin..., ou plus souvent chez des enfants tels les jeunes Meh..., Michel Br..., l'enfant Dye..., et Franck Heu...

$2^{\circ}$ Les enfants présentent des lésions le plus souvent banales, soit de teigne tondante microsporique, soit d'herpès circinés du tronc, du visage ou des membres; les deux types de lésions peuvent coexister chez le même enfant.

Les adultes présentent des herpès circinés siégeant surtout sur les parties découvertes ou, dans certains cas particuliers, sur tout le corps (ex. $\mathbf{M}^{\mathrm{me}}$ Fer... qui laissait son chat dormir sur son lit).

$3^{\circ}$ Un contage animal a été retrouvé assez souvent.

Il a été bien précisé pour la famille Bar..., l'enfant Dye..., Michel Bre..., la famille Lac..., où un chat a été incriminé, mais sans que la preuve biologique ait pu être faite par un prélèvement.

Un contage très précis a été mis en évidence pour la famille Thi... où le chat a pu être examiné sous lampe de Wood. Dans le cas de $\mathbf{M}^{\mathrm{m} \bullet}$ Fer..., on a pu également obtenir des poils du chat suspecté, les examiner et les ensemencer.

Mais dans plusieurs cas, la notion de contage reste imprécise ou inexistante.

$4^{\circ}$ Du point de vue de la répartition géographique, signalons une famille contaminée en Savoie (famille Lac...). Les autres malades habitent tous Paris ou ses environs immédiats. Un chat contaminateur provient d'un refuge de Gennevilliers, deux autres chats ont été achetés à Paris même dans des magasins spécialisés dans la vente des petits animaux. 


\begin{tabular}{|c|c|c|c|c|}
\hline $16 / 9 / 66$ & Enfant Meh... & & $\begin{array}{l}\text { Teigne tondante micros- } \\
\text { porique }\end{array}$ & Chat ? \\
\hline $15 / 12 / 66$ & $\begin{array}{l}\text { Michel Bre... } \\
10 \text { ans }\end{array}$ & 93 - Bagnolet & $\begin{array}{l}\text { Teigne tondante rétro- } \\
\text { auriculaire }\end{array}$ & Chat \\
\hline $29 / 3 / 67$ & $\begin{array}{l}\text { Antony Co... } \\
8 \text { ans } 1 / 2 \\
\text { Mme Co... } \\
50 \text { ans }\end{array}$ & $\begin{array}{l}78 \text { - La Celle- } \\
\text { St-Cloud }\end{array}$ & $\begin{array}{l}\text { H. circiné du cou, quel- } \\
\text { ques squames sur la } \\
\text { nuque } \\
\text { Rechute fin juillet : tei- } \\
\text { gne tondante évoluant } \\
\text { vers kérion } \\
\text { H. circiné du visage en } \\
\text { août }\end{array}$ & $\begin{array}{l}\text { Chat disparu Noël } \\
66 \\
\text { Contacts épisodi- } \\
\text { ques avec chien sup- } \\
\text { posé sain }\end{array}$ \\
\hline $12 / 9 / 67$ & $\begin{array}{l}\text { !oëlle Bar... } \\
7 \text { ans } \\
\text { Une sœur } 5 \text { ans } \\
\text { Un frère } 12 \text { ans } \\
\text { Un frère } 9 \text { ans }\end{array}$ & $\begin{array}{l}\text { 93- Neuilly- } \\
\text { Plaisance }\end{array}$ & $\begin{array}{l}\text { H. circinés } \\
\text { H. circinés } \\
\text { H. circinés } \\
\text { H. circinés }\end{array}$ & $\begin{array}{l}\text { Chat \& de race», } \\
\text { gardé trois semaines } \\
\text { avant }\end{array}$ \\
\hline $25 / 10 / 67$ & $\begin{array}{l}\text { Catherine Thi... } \\
10 \text { ans } \\
\text { Bernard Thi... } \\
9 \text { ans } \\
\text { Philippe Thi... } \\
7 \text { ans } \\
\text { Mme Thi... } \\
34 \text { ans }\end{array}$ & 92 - Nanterre & $\begin{array}{l}\text { H. circinés du visage } \\
\text { H. circiné du nez en } \\
\text { voie de guérison sponta- } \\
\text { née } \\
\text { H. circiné de l'abdomen } \\
\begin{array}{l}\text { H. circinés de lavant- } \\
\text { bras et du dos }\end{array} \\
\end{array}$ & $\begin{array}{l}\text { Jeune chat recueilli } \\
\text {.epuis } 2 \text { mois Wood, } \\
\text { ED +, Cult. + (1) }\end{array}$ \\
\hline $7 / 11 / 67$ & $\begin{array}{l}\text { Mlle Bin... } \\
23 \text { ans }\end{array}$ & Paris-15 & $\begin{array}{l}\text { H. circiné de l'avant- } \\
\text { bras }\end{array}$ & Chien? \\
\hline $22 / 11 / 67$ & $\begin{array}{l}\text { Annick Eud... } \\
5 \text { ans }\end{array}$ & Paris-8 ${ }^{\circ}$ & $\begin{array}{l}\text { Teigne tondante micros- } \\
\text { porique }\end{array}$ & Chat? \\
\hline $1 / 12 / 67$ & $\begin{array}{l}\text { Mme Ingrid Fer.. } \\
19 \text { ans }\end{array}$ & Paris-17 & $\begin{array}{l}\text { H. circinés de l'épaule, } \\
\text { du sein gauche, du dos } \\
\text { Le mari aurait les mê- } \\
\text { mes lésions. }\end{array}$ & $\begin{array}{l}\text { Chat acheté un mois } \\
\text { avant dans une oi- } \\
\text { sellerie, Wood +. } \\
\text { ED +, Cult. + }\end{array}$ \\
\hline $7 / 12 / 67$ & $\begin{array}{l}\text { Enfant Dye... } \\
7 \text { ans }\end{array}$ & 92 - Meudon & $\begin{array}{l}\text { Teigne tondante du } \\
\text { cuir chevelu et } \mathrm{H} \text {. cir- } \\
\text { ciné sur sourcils }\end{array}$ & $\begin{array}{l}\text { Chat acheté peu } \\
\text { avant dans un ma- } \\
\text { gasin }\end{array}$ \\
\hline $26 / 12 / 67$ & $\begin{array}{l}\text { Juan Gar... } \\
9 \text { ans } 1 / 2 \\
\text { Emmanuel Gar... } \\
8 \text { ans } 1 / 2 \\
\text { Stéphane Gar... } \\
5 \text { ans }\end{array}$ & & $\begin{array}{l}\text { Teigne tondante de la } \\
\text { tempe, occiput. et } \mathrm{H} \text {. } \\
\text { circinés multiples } \\
\text { Teigne tondante fron- } \\
\text { tale } \\
\text { Teigne tondante occipi- } \\
\text { tale } \\
\text { Apparition sous griséo- } \\
\text { fulvine de plusieurs her- } \\
\text { pès circinés le } 22 / 1 / 68 \\
\text { et le } 5 / 2 / 68 \\
\end{array}$ & Chien, Wood - \\
\hline $20 / 1 / 68$ & $\begin{array}{l}\text { Franck Heu... } \\
7 \text { ans }\end{array}$ & $\begin{array}{l}92 \text { - Courbe- } \\
\text { voie }\end{array}$ & Teigne tondante & Chat? \\
\hline $13 / 2 / 68$ & $\begin{array}{l}\text { Sylvie Lac... } \\
9 \text { ans } \\
\text { Véronique Lac... } \\
7 \text { ans } \\
\text { Sophie Lac... } \\
6 \text { ans }\end{array}$ & & $\begin{array}{l}\text { Teigne tondante } \\
\text { Teigne tondante } \\
\text { Herpès circinés du } \\
\text { tronc }\end{array}$ & $\begin{array}{l}\text { Chat en Savoie; le } \\
\text { chat de la famille } \\
\text { reste indemne }\end{array}$ \\
\hline
\end{tabular}

(1) $\mathrm{ED}=$ Examen direct. 
$5^{\circ}$ L'évolution sous traitement est le plus souvent favorable.

Pour les teignes tondantes, la griséofulvine associée à la désinfection locale et au rasage a entraîné la guérison dans un délai moyen de deux mois. Une exception pourtant doit être signalée : l'enfant Co..., débile mental, dont les lésions d'herpès circinés du cou et les lésions squameuses diffuses de la nuque ont guéri rapidement par un traitement local et général, mais qui vit secondairement apparaître une teigne évoluant vers un très important kérion pariétal.

Pour les herpès circinés, soulignons la possibilité de guérison spontanée (cas de Bernard Thi...) et l'évolution favorable très rapide sous traitement local dans presque tous les cas. On a vu cependant apparaître, au cours du traitement d'une teigne tondante par griséofulvine, des herpès circinés du cou et du visage, puis des cuisses (Stéphane Gar...).

\section{Etude mycologique.}

Aucun argument particulier ne peut être tiré de l'examen direct qui montre des filaments mycéliens dans les squames et un parasitisme microsporique des cheveux.

L'examen sous lampe de Wood a toujours montré dans les cas de teignes tondantes la fluorescence verte habituelle des microspories.

La vitesse de pousse sur les milieux de Sabouraud classiques ne diffère pas sensiblement de celle des souches typiques de Microsporum canis; mais l'aspect macroscopique des cultures est d'emblée très différent. Il s'agit d'une culture poudreuse ou très finement duveteuse, au ras de la gélose, avec parfois quelques plis radiés sinueux partant du centre de la colonie qui a tendance à se déprimer légèrement. En surface, la colonie est d'un blanc très pur. L'envers est de teinte chamois clair, sans pigment diffusant dans la gélose. Jamais on ne voit le pigment orangé caractéristique des souches habituelles de Microsporum canis. Lors des repiquages successifs, cet aspect macroscopique est resté remarquablement constant.

Toutes les souches ont pu être repiquées plusieurs fois sur gélose peptonée (gélose «conservation » de Sabouraud). Là encore, ces souches montrent un aspect remarquablement identique et semble-t-il constant : cultures poussant d'abord rapidement, puis d'extension ultérieure très lente, sous forme d'une colonie au ras de la gélose, plutôt poudreuse ou à duvet extrêmement court, avec des filaments immergés dépassant en périphérie la colonie de surface, de teinte un peu verdâtre. Le centre de la colonie tend à former une dépression d'où partent trois à cinq plis radiés peu profonds, assez rectilignes. L'envers de la colonie ne montre pas de pigment.

L'étude microscopique par dissociation d'un fragment de colonie ou par culture sur lame montre deux éléments essentiels :

$1^{\circ}$ D'assez nombreuses macroconidies dont la forme, la taille et l'aspect échinulé sont tout à fait comparables à ceux des souches de Microsporum canis habituelles. Tout au plus peut-on signaler que la paroi nous semble un peu moins épaisse. 


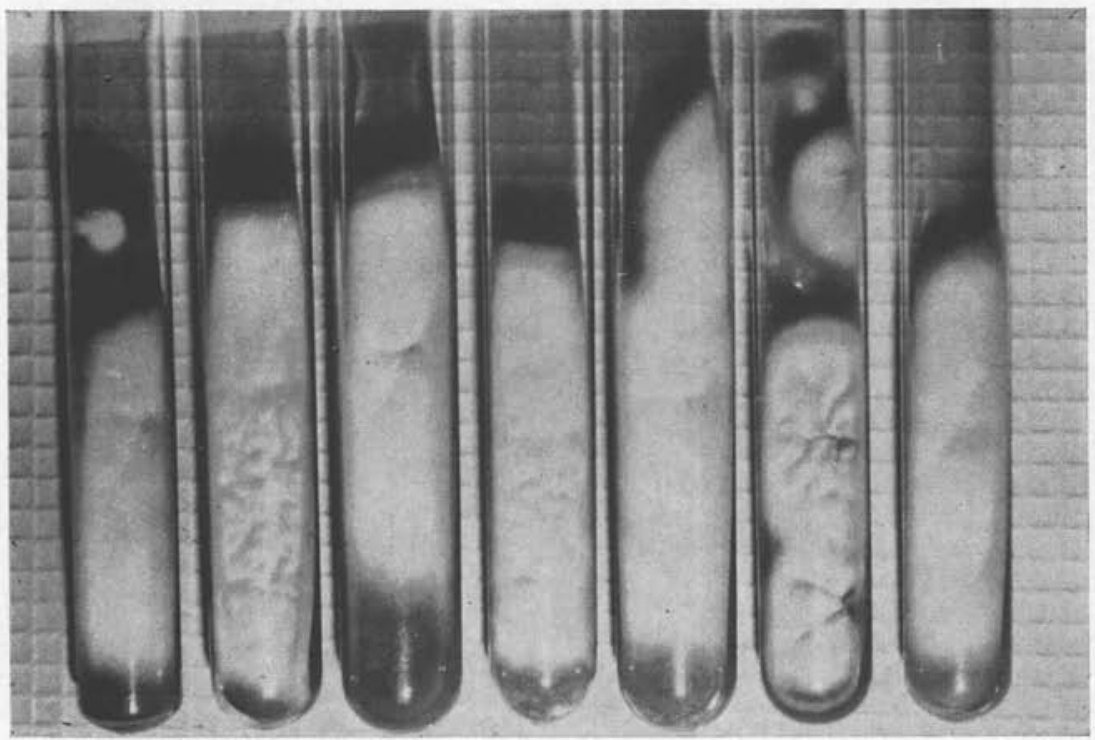

Fig. 1. - Cultures sur milieux de Sabouraud glucosés de 7 souches différentes de Microsporum canis var. pulverulentum datant de 15 jours

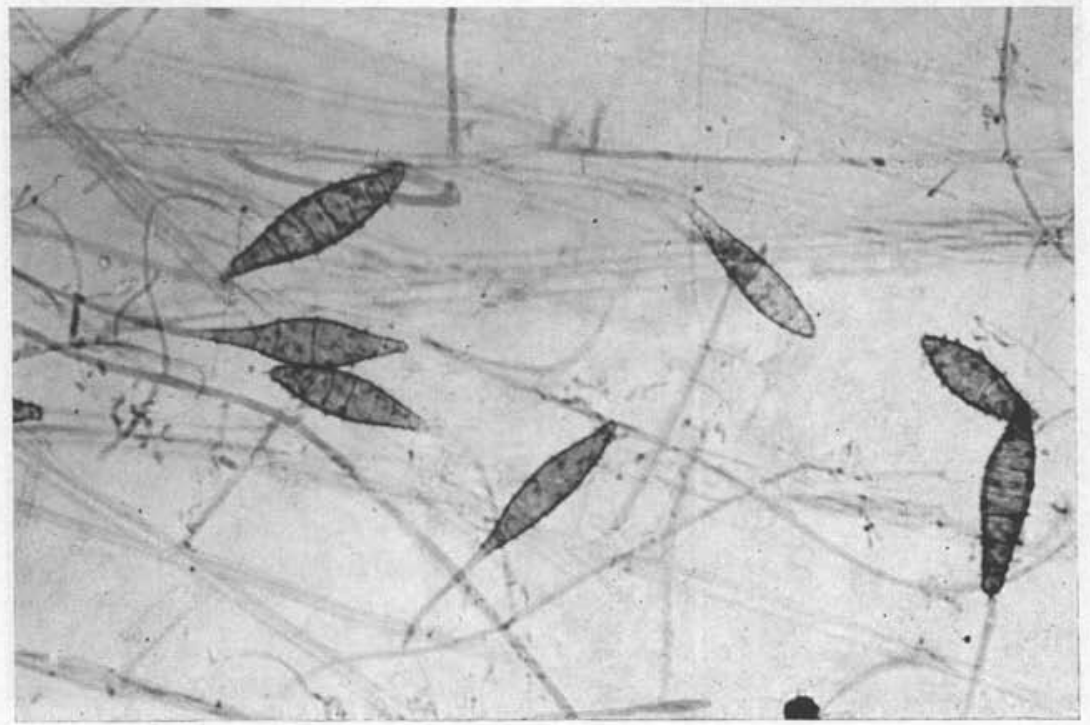

Fig. 2. - Dissociation dans le lactophénol d'une culture datant de 10 jours $=$ Fuseaux caractéristiques et nombreuses microconidies (objectif $\times 25$ ) 


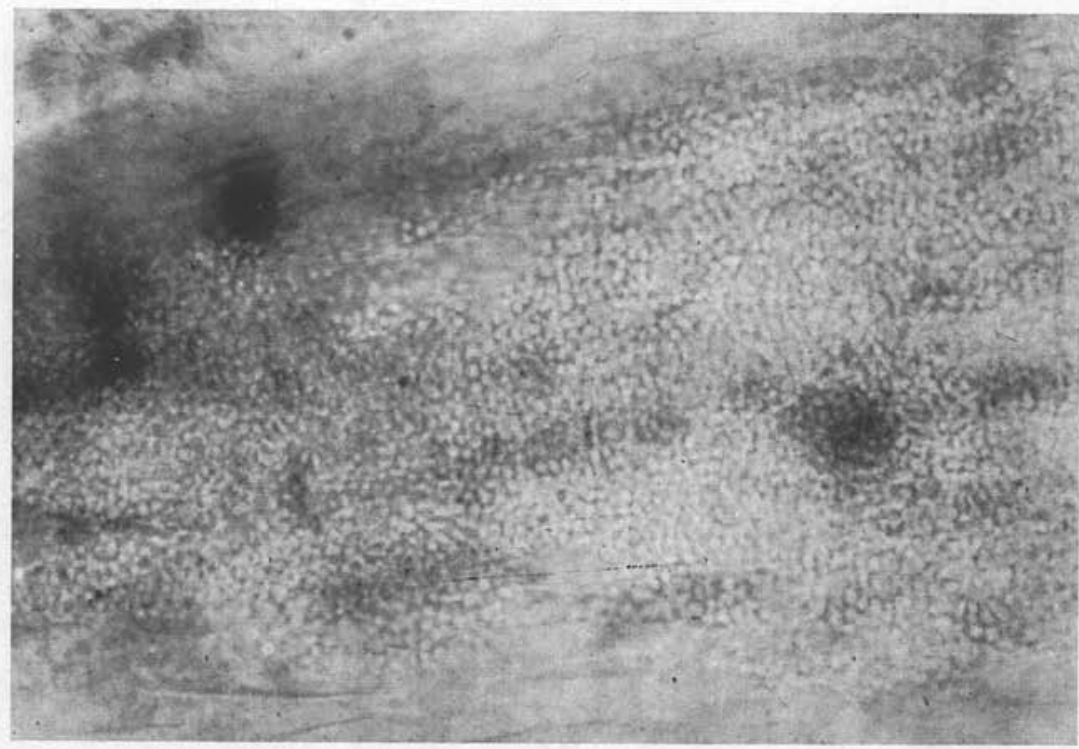

FIG. 3. - Examen direct dans le lactophénol d'un poil de chat spontanément parasité. Ce poil, fluorescent sous lampe de Wood montre une gaine périphérique de petites spores (objectif $\times 40$ )

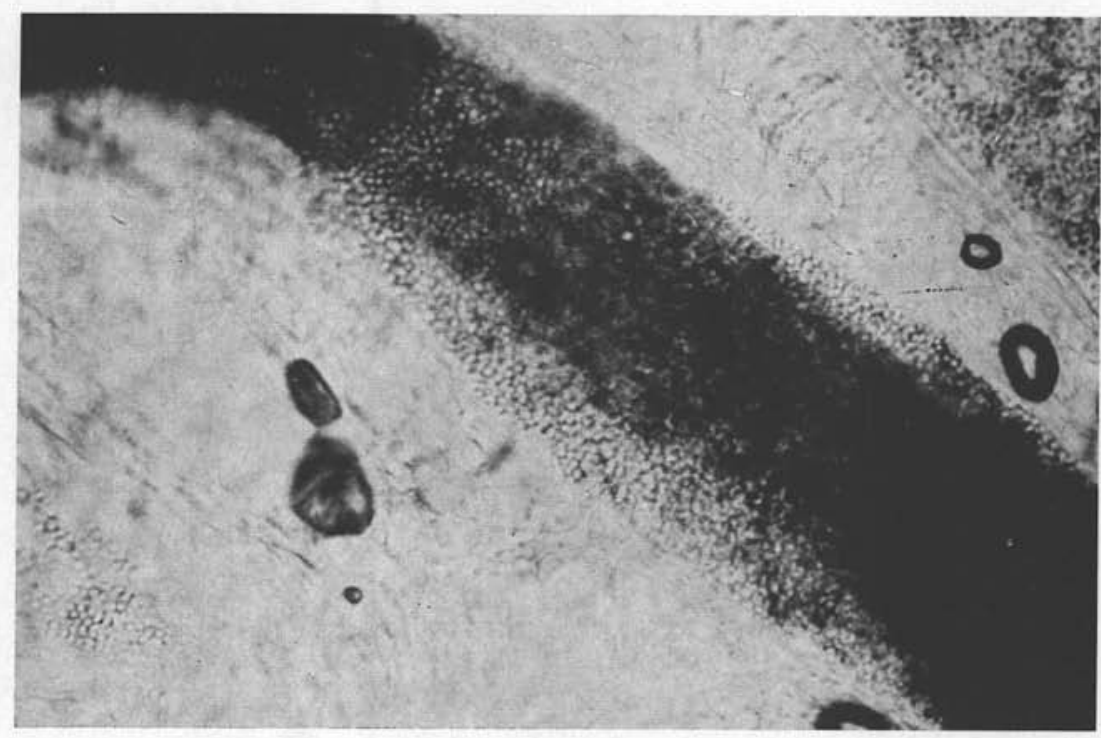

Fig. 4. - Examen direct dans le lactophénol d'un poil de cobaye inoculé avec un broyat de culture. La gaine microscopique est bien visible (objectif $\times 25$ ) 
$2^{\circ}$ D'innombrables microconidies piriformes ou en forme de bâtonnets. Certains rameaux montrent une disposition de type Acladium; en d'autres points, l'abondance des microconidies réalise de véritables buissons cachant les filaments mycéliens.

Ces deux éléments semblent demeurer constants lors des repiquages jusqu'ici pratiqués. On n'a pas observé de pléomorphisation des colonies.

Toutes les souches ont été inoculées à des cobayes. L'évolution de la maladie expérimentale a été la même dans tous les cas : vers le cinquième jour, apparition de quelques squames; vers le huitième jour, on constate sous lampe de Wood la présence de poils fluorescents, non cassés ; au quinzième jour, on observe un tapis de poils cassés, fluorescents, avec des squames abondantes. L'aspect est parfois un peu inflammatoire. Cet aspect persiste jusque vers le $20-22^{\circ}$ jour et l'évolution se fait vers la guérison spontanée. L'examen direct des poils de ces cobayes montre un parasitisme de type microsporique ; les cultures de ces poils nous ont permis d'obtenir à nouveau les souches initiales.

\section{Discussion.}

$1^{\circ} \mathrm{Du}$ point de vue clinique, cet ensemble assez disparate d'observations rappelle tout à fait la clinique touffue du Microsporum canis habituellement isolé dans la région parisienne.

$2^{\circ} \mathrm{Du}$ point de vue mycologique, bien qu'il soit maintenant difficile de se faire une idée précise de l'aspect de souches fraîches de Microsporum audouini, qui a maintenant totalement disparu de la région parisienne, il semble que l'aspect macroscopique de nos souches soit très proche de celui de ce parasite. Et cela est vrai au point que les premières identifications des souches que nous étudions ici ont été rendues sous l'appellation de Microsporum audouini. C'est la notion de contact avec un chat, d'abord imprécise, puis l'examen du chat contaminateur de la famille Thi... qui nous ont amenés à revoir ces déterminations et à regrouper alors l'ensemble de ces souches.

L'individualité de l'aspect microscopique nous paraît bien établie ; il est constant lors des repiquages successifs et très comparable d'une souche à l'autre. Les macroconidies sont celles du Microsporum canis, mais leur nombre est bien moindre ; d'autre part, elles sont bien plus nombreuses que celles du Microsporum audouini qui sont en outre souvent moins bien formées. L'abondance extrême des microconidies que nous observons ici ne se voit ni dans les souches classiques de Microsporum audouini, ni surtout dans les souches de Microsporum canis.

En ce qui concerne le pouvoir pathogène expérimental, la maladie que nous avons provoquée chez le cobaye semble superposable à celle provoquée par Microsporum canis. Rappelons que par deux fois ces souches atypiques ont été isolées chez des chats contaminateurs.

Nous proposons le nom de Microsporum canis pulverulentum n. s./sp., étant bien entendu qu'il serait abusif d'en faire une espèce distincte. 
Microsporum canis Bodin sub-species pulverulentum nova differt a forma typica coloratione pallente mycelii in culturis quae nunquam sunt flavae neque colore aurantii et microscopice, a multis microconidiis et a rarioribus macroconidiis. Haec sub-species aeque est inoculata a felibus in humanam speciem et reperta est copiose in regione Lutetiae.

Il semble donc bien s'agir d'une sous-espèce de Microsporum canis devenue subitement fréquente dans la région parisienne. Peut-être même existe-t-elle dans la France entière puisque la famille Gar... a été contaminée en Savoie. Nous avons vu que ces souches représentaient à peu près le $1 / 8^{\circ}$ de nos cas de microspories au cours de ces 18 derniers mois. Le rôle non négligeable des magasins de vente de petits animaux, qui ne sont soumis à aucun contrôle vétérinaire, a été mis en évidence là encore, tout comme on le retrouve dans la transmission du Microsporum canis classique.

Il y a lieu d'insister pour terminer sur l'intérêt pratique de cette sous-espèce qui dépasse à notre sens l'intérêt mycologique pur en raison de sa confusion inévitable avec un Microsporum audouini si un contrôle microscopique attentif n'est pas effectué. Cette erreur risque d'orienter la prophylaxie dans une mauvaise direction; et l'on doit savoir maintenant que la recherche d'une contamination animale doit être faite avec soin, même quand la culture n'en impose nullement à priori pour un Microsporum canis.

\section{Bibliographie}

Bodin (E.) et Almy (J.). - Le Microsporum du chien. Recueil de médecine vétérinaire, 15 mars 1897.

Conant (N. F.), Smith (D. T.), Baker (R. D.), Callaway (J. L.), Martin (D. S.), 1954. Manual of Clinical mycology. W. B. Saunders Company, Philadelphia and London.

COUdeRT (J.), 1955. - Guide pratique de Mycologie médicale. Masson édit.

Emmons (C. W), Binford (C. H.), UtZ (J.-P.), 1964. - Medical Mycology. Lea and Febiger. Philadelphia.

Fox (C.) et BlaxalL, 1896. - Note on two cases of tinea circinata. British Journal of Dermatology. T. X, $\mathrm{n}^{\circ} 112$.

Hildick-Smith (G.), Blank (H.), Sarkany (L.), 1964. - Fungus diseases and their treatment. Little, Brown and Company, Boston.

Rivalier (E.), 1936. - Nouvelle pratique dermatologique. Masson édit. Paris.

Sabouraud (R.), 1910. - Maladies du cuir chevelu, les teignes. Masson édit. Paris.

VANBREUSEGHEM (R.), 1966. - Guide pratique de Mycologie médicale et vétérinaire. Masson édit. Paris. 